\title{
Noise effects for the Depolarizing Channel
}

\author{
Julian Juhi-Lian Ting \\ jlting@multimania.com
}

(November 6, 2018)

\begin{abstract}
The possibility of stochastic resonance of a quantum channel and hence the noise enhanced capacity of the channel is explored by considering the depolarizing channel. The fidelity of the channel is also considered. Although there is no clear evidence for noise enhanced capacity found, there is enhancement for the fidelity for the depolarizing channel.
\end{abstract}

\section{INTRODUCTION}

Recently, because of the development of quantum computers [1] people have become interested in information transmission thorough quantum channels 22. Quantum information theories can be used to describe processes such as data storage, quantum cryptography [3], and quantum teleportation [4]. However, after an initial burst of papers following Shor's discovery of quantum factoring algorithm [5], almost every work is aiming to solve the decoherence problem. There are people using NMR techniques, which provide longer decoherence time than previous techniques, claiming they can built a quantum computer with a cup of coffee [6]. There are also people trying to use various software methods, in particular, quantum error correcting codes, to correct decoherence induced errors. Apparently, decoherence is a hurdle need to be surmounted before quantum computers can be materialized. However, is decoherence, the counterpart of classical noise, really nuisance? For people who know stochastic resonance, the answer is perhaps 'no'.

Stochastic resonances in nonlinear dynamics is about noise enhancement of some useful properties of a system. It have been considered for both quantum and classical periodical signals [7]. The consideration for aperiodical signal cases is rather recent [8,9]. However, most works are concerned about classical channels. Therefore, the consideration for quantum channels is of interest in itself whether there is noise enhancement or not, because it is a missing piece in the theories of stochastic resonance.

In the periodical cases spectral properties of a system are generally used to characterize their performance. However, it has been recognized recently that spectral properties of a system are adequate for describing the system only when the system is linear [10]. Mutual information, a kind of entropy change in statistical mechanics, seems to be an even better parameter to describe the resonance. In the works for classical aperiodical stochastic resonances the mutual information between the input and the output of the channel has been used as a measure of channel performance to be enhanced by the noise. A peak in the mutual information versus noise curve indicate resonance. To study the noise enhanced channel capacities of quantum channels one need a measure for noise and a measure for the channel capacities, or a measure for any other property interested. The problem is: which quantity can be used as the correct measure?

In what follows the capacity and fidelity versus noise relation of the depolarizing channel [1] is considered. The capacity versus flipping rate relation of the depolarizing channel has been previously considered by Lloyd [12]. However, it is the capacity versus noise relation, which has its interpretation in stochastic resonance, is of more importance in physics. A similar consideration has been presented for the two-Pauli channels by Ting [13]. This paper therefore has three folds of purposes: Firstly, a more through explanation of the problem considered than the previous paper is taken. Secondly, we tried to establish links with previous works in quantum computing, in particular those related to Lloyd's, and in other fields of physics [14]. Thirdly, we tried to explore one more channel.

\section{THE NOISY CHANNEL MODEL}

Schumacher et al. [15] have developed a quantum information theory. In their formulation a quantum channel can be considered as a process defined by an input density matrix $\rho_{x}$, and an output density matrix $\rho_{y}$, with the process described by a quantum operation, $\mathcal{N}$,

$$
\rho_{x} \stackrel{\mathcal{N}}{\rightarrow} \rho_{y} .
$$


Because of decoherence, the super-operator $\mathcal{N}$ is not unitary. However, on a larger quantum system that includes the environment $E$ of the system, the total evolution operator $U_{x E}$ become unitary. This environment may be considered to be initially in a pure state $\left|0_{E}\right\rangle$ without loss of generality. In this case, the super-operator can be written as

$$
\mathcal{N}\left(\rho_{x}\right)=\operatorname{Tr}_{E} U_{x E}\left(\rho_{x} \otimes\left|0_{E}\right\rangle\left\langle 0_{E}\right|\right) U_{x E}^{\dagger} .
$$

The partial trace, $\operatorname{Tr}_{E}$, is taken over environmental degree of freedom. Eq. (2) can be rewritten as a completely positive linear transformation acting on the density matrix:

$$
\mathcal{N}\left(\rho_{x}\right)=\sum_{i} A_{i} \rho_{x} A_{i}^{\dagger}
$$

in which the $A_{i}$ satisfy the completeness relation

$$
\sum_{i} A_{i}^{\dagger} A_{i}=I
$$

which is equivalent to requiring $\operatorname{Tr}\left[\mathcal{N}\left(\rho_{x}\right)\right]=1$. Conversely, any set of operators $A_{i}$ satisfying Eq. (何) can be used in Eq. (3) to give rise to a valid noisy channel in the sense of Eq. (21). Eq. (3) is in fact a Schrödinger evolution of the density matrix.

In order to study the noise effects, we certainly need a definition of noise. Schumacher has also gave a definition of noise, i.e.

$$
N\left(\rho_{x}, \mathcal{N}\right) \equiv-\operatorname{Tr}\left(W \log _{2} W\right)
$$

with

$$
W_{i j} \equiv \operatorname{Tr}\left(A_{i} \rho_{x} A_{j}^{\dagger}\right) .
$$

It measures the amount of information exchanged between the system $x$ and the environment $E$ during their interaction [2]. If the environment is initially in a pure state, the entropy exchange is just the environment's entropy after the interaction.

The next quantity we need is a measure of any quantity we are interested in. For a communication channel the most important quantities are its capacity and its fidelity. Although the coherence information is generally believed to represent only a lower bound on the channel capacity in the Shannon's definition, it can be used to represent the channel capacity without talking about encoding 16, 17. It is defined as

$$
C\left(\rho_{x}, \mathcal{N}\right) \equiv H\left(\mathcal{N}\left(\rho_{x}\right)\right)-N\left(\rho_{x}, \mathcal{N}\right),
$$

in which $H(\bullet)=-\operatorname{Tr}\left[\bullet \log _{2} \bullet\right]$ is the von Neumann entropy [18]. Coherent information plays a role in quantum information theory analogous to that played by the mutual information in classical information theory. It can be positive, negative, or zero.

\section{THE DEPOLARIZING CHANNEL}

A depolarizing channel can be written in terms of $A_{i}$ 's in Eq. (3) as

$$
A_{1}=\sqrt{x} I, \quad A_{2}=\sqrt{\frac{(1-x)}{3}} \sigma_{1}, \quad A_{3}=\sqrt{\frac{(1-x)}{3}} \sigma_{3}, \quad A_{4}=-i \sqrt{\frac{(1-x)}{3}} \sigma_{2},
$$

where $I$ is the identity matrix and $\sigma_{1}, \sigma_{2}$, and $\sigma_{3}$ are the Pauli matrices, i.e.,

$$
\sigma_{1}=\left(\begin{array}{rr}
0 & 1 \\
1 & 0
\end{array}\right), \quad \sigma_{2}=\left(\begin{array}{rr}
0 & -i \\
i & 0
\end{array}\right), \quad \sigma_{3}=\left(\begin{array}{rr}
1 & 0 \\
0 & -1
\end{array}\right) .
$$

This channel can be interpreted as: with probability $x$, it leaves the qubit alone; with probability $(1-x) / 3$ it either flip the qubit amplitude $\left(A_{2}\right)$, or rotate the qubit $\left(A_{3}\right)$, or do both flip and rotation to the qubit $\left(A_{4}\right)$. In other words, if one has a quantum state

$$
|\Psi\rangle=\alpha|0\rangle+\beta|1\rangle
$$


goes through such a channel, the resulting change will be

$$
\begin{aligned}
& |\Psi\rangle \stackrel{x}{\longrightarrow}|\Psi\rangle, \\
& |\Psi\rangle \stackrel{(1-x) / 3}{\longrightarrow} \sigma_{1}|\Psi\rangle=\alpha|1\rangle+\beta|0\rangle, \\
& |\Psi\rangle \stackrel{(1-x) / 3}{\longrightarrow} \sigma_{3}|\Psi\rangle=-\alpha|0\rangle+\beta|1\rangle, \\
& |\Psi\rangle \stackrel{(-x) / 3}{\longrightarrow} \sigma_{1} \sigma_{3}|\Psi\rangle=-\alpha|1\rangle+\beta|0\rangle .
\end{aligned}
$$

It is similar to the two-Pauli channel considered previously [13], except the depolarizing channel flips the qubit with equal probability for all three Pauli operators.

A general (input) state in the Bloch sphere representation can be written as

$$
\rho_{x}=\frac{1}{2}(I+\vec{a} \cdot \vec{\sigma}) .
$$

Here, $\vec{a}=\left(a_{1}, a_{2}, a_{3}\right)$ is the Bloch vector of length unity or less, and $\vec{\sigma}$ is the vector of Pauli matrices. The action of the channel on this density state is:

$$
\mathcal{N}\left(\rho_{x}\right)=\frac{1}{2}(I+\vec{b} \cdot \vec{\sigma})
$$

in which

$$
\vec{b}=\left(\frac{4}{3} x-\frac{1}{3}\right)\left(a_{1}, a_{2}, a_{3}\right) .
$$

It is clear from this expression that the whole Bloch vector is shrunken by a factor of $(4 x-1) / 3$.

The logarithm of a density matrix is defined in its orthogonal basis, i.e., pure state decomposition [19]. If $\rho_{x}$ can be written as

$$
\rho_{x}=\sum_{i} a_{i}|i\rangle\langle i|
$$

then the logarithm of $\rho_{x}$ is given by

$$
\log \rho_{x}=\sum_{i} \log a_{i}|i\rangle\langle i| .
$$

The von Neumann entropy can be shown to become

$$
H\left(\rho_{x}\right)=-\sum_{i} a_{i} \log a_{i}
$$

in which $a_{i}$ s are the eigenvalues of the density matrix $\rho_{x}$. An eligible density matrix never have negative or imaginary eigenvalue.

The matrix $W$ for the depolarizing channel read,

$$
W=\left(\begin{array}{cccc}
x & a_{1} \sqrt{\frac{x(1-x)}{3}} & a_{3} \sqrt{\frac{x(1-x)}{3}} & i a_{2} \sqrt{\frac{x(1-x)}{3}} \\
a_{1} \sqrt{\frac{x(1-x)}{3}} & \frac{1-x}{3} & \frac{i a_{2}(1-x)}{3} & \frac{a_{3}(1-x)}{3} \\
a_{3} \sqrt{\frac{x(1-x)}{3}} & \frac{-i a_{2}(1-x)}{3} & \frac{1-x}{3} & \frac{a_{1}(1-x)}{3} \\
-i a_{2} \sqrt{\frac{x(1-x)}{3}} & \frac{a_{3}(1-x)}{3} & \frac{a_{1}(1-x)}{3} & \frac{1-x}{3}
\end{array}\right) .
$$

It has four eigenvalues, namely $\lambda_{1,2}=(1-x)(1 \pm|a|) / 3$ and $\lambda_{3,4}=\left[(1+2 x) \pm \sqrt{12 x(x-1)\left(1-|a|^{2}\right)+(1+2 x)^{2}}\right] / 6$, in which $|a|=\sqrt{a_{1}^{2}+a_{2}^{2}+a_{3}^{2}}$. Hence,

$$
N=-\sum_{i=1}^{4} \lambda_{i} \log _{2} \lambda_{i}
$$


while

$$
H\left(\rho_{y}\right)=-\sum_{i=1}^{2} \theta_{i} \log _{2} \theta_{i},
$$

with $\theta_{1,2}=[3 \pm(1-4 x)|a|] / 6$. This $x-N, C-N$ relationship is plotted in Fig. 1. Similar enhancements like the two-Pauli channel considered previously are found [13]. There are clear evidences of noise enhancement for the fidelity. In order to compare with previous authors the coherent information versus flipping rate they are also plotted in the figure.

Furthermore, for a communication channel the (entangled) fidelity,

$$
F=\sum_{\mu}\left(\operatorname{Tr} \rho_{x} A_{\mu}\right)\left(\operatorname{Tr} \rho_{x} A_{\mu}^{\dagger}\right)
$$

is also of our concern, since it represent the quality of the signal transmitted. For the depolarizing channel

$$
F=\frac{1}{3}|a|^{2}(1-x)+x
$$

We found not only for the capacity but also for for the noise and for the fidelity, $a_{1}, a_{2}$ and $a_{3}$ are on the same footing. The channel is symmetric for the exchange of $a_{1}, a_{2}$ and $a_{3}$. Only the length of the vector $\vec{a}$ matters. The relation between the fidelity and the noise is plotted with the coherent information in Fig. 1. Note that some people might think fidelity can be used as a measure of the noise strength. However, it is only an indirect measure. It measures the effect of the noise instead of the noise itself.

\section{CONCLUSION}

In summary, a quantum stochastic resonance like scenario in a quantum communication channel is considered. It is important to note here that which definition of a parameter of the system is a suitable one is depending upon the problem one would like to ask. Within the context of stochastic resonance there is no right or wrong of the parameters used. In stochastic resonance we can look for any definition we like for the resonance. In this paper we have demonstrated two.

Gisin and Wolf said quantum cryptography lies at the intersection of two of the major sciences of the 20th century: quantum mechanics and information theory 20. The present work is at a three-junction of modern sciences, namely, stochastic resonances, quantum mechanics, and information theories.

Other effects in quantum communication are under further consideration.

I thank Mrs. L. L. Harn, the librarian of NCHU physics department, for providing me the computing facility and the library access.

[1] A. Steane, Rep. Prog. Phys., 61, 117 (1998).

[2] B. Schumacher, Phys. Rev. A 54, 2614 (1996).

[3] D. Deutsch, A. Ekert, R. Jozsa, C. Macchiavello, S. Popescu and A. Sanpera, Phys. Rev. Lett. 77, 2818 (1996).

[4] C. H. Bennett, G. Brassard, S. Popescu, B. Schumacher, J. A. Smolin and W. K. Wootters, Phys. Rev. Lett. 76, 722 (1996).

[5] P. W. Shor, in Proceedings of the 35th Annual Symposium on Foundations of Computer Science, (IEEE Computer Society Press, New York, 1994), p124.

[6] N. A. Gershenfeld and I. L. Chuang, Science, 275, 350 (1997).

[7] A. Bulsara and L. Gammaitoni, Phys. Today, 49, 39 (1996).

[8] A. R. Bulsara and A. Zador, Phys. Rev. E, 54, 2185 (1996).

[9] F. Chapeau-Blondeau, Phys. Rev. E, 55, 2016 (1997).

[10] J. W. C. Robinson, D. E. Asraf, A. R. Bulsara and M. E. Inchiosa, Phys. Rev. Lett., 81, 2850 (1998).

[11] P. W. Shor and J. A. Smolin, quant-ph/9604006.

[12] S. Lloyd, Phys. Rev. A, 55, R1613, quant-ph/9604015 (1997). 
[13] J. J.-L. Ting, Phys. Rev. E, 592801 (1999).

[14] J. Preskill, quant-ph/9904022.

[15] B. Schumacher and M. A. Nielsen, Phys. Rev. A, 54, 2629 (1996).

[16] H. Barnum, J. A. Smolin and B. M. Terhal, Phys. Rev. A, 58, 3496 (1998).

[17] H. Barnum, E. Knill and M. A. Nielsen, IEEE Trans. Info. Theory, quant-ph/9809010, (1998).

[18] J. von Neumann, Mathematical Foundations of Quantum Mechanics, translated by E. T. Beyer (Princeton University Press, Princeton, 1955).

[19] A. Peres, Quantum Theory: Concepts and Methods, (Kluwer Academic Publisher, 1993), page 68.

[20] N. Gisin and S. Wolf, quant-ph/9902048.

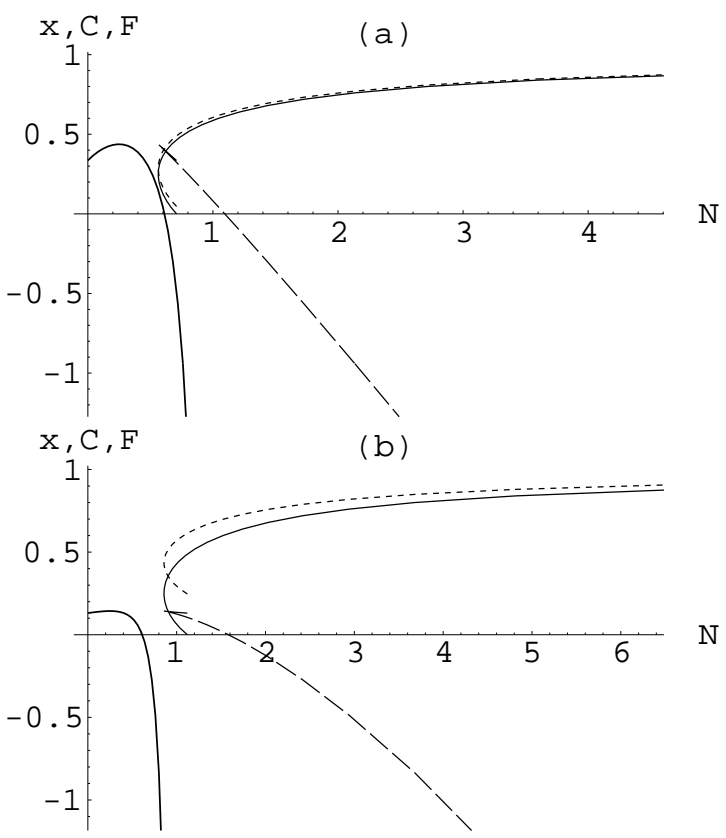

FIG. 1. Parametric plots of the retention rate, $x$, versus noise, $N$ (solid lines); coherence information, C, versus noise, $\mathrm{N}$, (long dashed lines); fidelity, F, versus noise, N, (short dashed lines) and coherent information, C, versus flipping rate, $\mathrm{x}$, (thick solid line). for the parameter $x$ from 0 to 1 and initial states: (a) $a_{1}=1 / 10, a_{2}=2 / 10, a_{3}=3 / 10 ;(\mathrm{b})$ $a_{1}=8 / 10, a_{2}=3 / 10, a_{3}=1 / 10$. 